\title{
State-Level Lifetime Medical and Work-Loss Costs of Fatal Injuries - United States, 2014
}

\author{
Feijun Luo, $\mathrm{PhD}^{1}$; Curtis Florence, $\mathrm{PhD}^{1}$
}

Injury-associated deaths have substantial economic consequences in the United States. The total estimated lifetime medical and work-loss costs associated with fatal injuries in 2013 were $\$ 214$ billion (1). In 2014, unintentional injury, suicide, and homicide (the fourth, tenth, and seventeenth leading causes of death, respectively) accounted for 194,635 deaths in the United States (2). In 2014, a total of 199,756 fatal injuries occurred in the United States, and the associated lifetime medical and work-loss costs were $\$ 227$ billion (3). This report examines the state-level economic burdens of fatal injuries by extending a previous national-level study (1). Numbers and rates of fatal injuries, lifetime costs, and lifetime costs per capita were calculated for each of the 50 states and the District of Columbia (DC) and for four injury intent categories (all intents, unintentional, suicide, and homicide). During 2014, injury mortality rates and economic burdens varied widely among the states and DC. Among fatal injuries of all intents, the mortality rate and lifetime costs per capita ranged from 101.9 per 100,000 and $\$ 1,233$, respectively (New Mexico) to 40.2 per 100,000 and $\$ 491$ (New York). States can engage more effectively and efficiently in injury prevention if they are aware of the economic burden of injuries, identify areas for immediate improvement, and devote necessary resources to those areas.

The numbers of injury-associated deaths in each of the 50 states and DC in 2014 were obtained from the National Vital Statistics System, and state-level lifetime costs were obtained from the Web-based Injury Statistics Query and Reporting System database (3). Injury death rates were calculated using the U.S. Census Bureau's bridged race population estimates for 2014. Lifetime costs, which include lifetime medical and work-loss costs, were computed by multiplying the number of injury deaths by average costs of treating injuries and earnings in 2010, adjusted to 2014 prices. Medical costs were derived from various sources that measure the costs of transport, health care in multiple settings, including emergency departments, hospitals, and nursing homes, and examination by a coroner or medical examiner (4). Work-loss costs were developed using earnings data from the U.S. Census Bureau's Current Population Survey and life expectancy data from CDC's National Center for Health Statistics. Numbers

\section{INSIDE}

12 Prevalence of Perceived Food and Housing Security 15 States, 2013

16 State Laws Requiring Hand Sanitation Stations at Animal Contact Exhibits - United States, MarchApril 2016

19 Using National Inpatient Death Rates as a Benchmark to Identify Hospitals with Inaccurate Cause of Death Reporting - Missouri, 2009-2012

23 Guidance for Assessment of Poliovirus Vaccination Status and Vaccination of Children Who Have Received Poliovirus Vaccine Outside the United States

26 Vital Signs: Decrease in Incidence of DiabetesRelated End-Stage Renal Disease among American Indians/Alaska Natives — United States, 1996-2013

33 Notes from the Field: Pan-Resistant New Delhi Metallo-Beta-Lactamase-Producing Klebsiella pneumoniae - Washoe County, Nevada, 2016

34 Notes from the Field: Occupational Lead Exposures at a Shipyard — Douglas County, Wisconsin, 2016

35 Notice to Readers

36 QuickStats

Continuing Education examination available at http://www.cdc.gov/mmwr/cme/conted_info.html\#weekly.

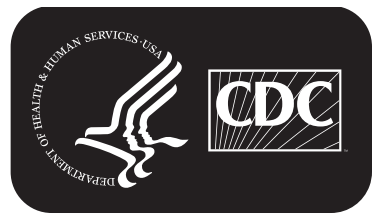

U.S. Department of Health and Human Services Centers for Disease Control and Prevention 
of deaths, rates, lifetime costs, and lifetime costs per capita (lifetime costs divided by the state population) were examined for each state and DC. Lifetime costs per capita were used for comparisons across states. Four intents of fatal injuries were considered: all intents, ${ }^{*}$ unintentional, suicide, and homicide. For each intent, state-level lifetime costs were estimated for the total population, for males and females, and for all intents. State-level lifetime costs were also estimated for three age groups: young (0-24 years), middle (25-64 years), and older ( $\geq 65$ years). State-level lifetime costs per capita were provided for the total population for each intent. In some state-intentpopulation combinations, average medical costs were statistically unstable, but these costs accounted for $<1 \%$ or $<5 \%$ of average lifetime costs. When both average medical costs and average work-loss costs were statistically unstable or when the mortality rates were unstable or missing, lifetime costs or lifetime costs per capita were not presented.

\section{Injuries from All Intents}

Injury mortality rates (per 100,000), lifetime costs (in 2014 U.S. dollars), and lifetime costs per capita (in 2014 U.S. dollars) varied widely among the 50 states and DC for each of the four intents. Overall, total injury-related mortality rate and lifetime costs per capita ranged from 101.9 per 100,000 and $\$ 1,233$, respectively (New Mexico) to 40.2 and $\$ 491$

\footnotetext{
*All intents category includes legal intervention and undetermined intent of injury, in addition to unintentional, suicide, and homicide.
}

(New York) (Table 1). The rates of overall male and female injury mortality were highest in New Mexico (141.1 and 63.7, respectively), and lowest in New York (58.9 and 23.1, respectively). New York also had the lowest injury mortality rate among persons aged $\geq 65$ years (87.1). The states with the highest and lowest lifetime fatal injury costs were California (\$20.9 billion) and Vermont (\$406 million), respectively. California had the highest number of injury deaths $(18,152)$ and DC the lowest number of injury deaths (385). The lifetime costs per capita for injuries of all intents ranged from $\$ 491$ to $\$ 1,233$ (Figure). The five states with the highest lifetime fatal injury costs per capita were New Mexico $(\$ 1,233)$, West Virginia $(\$ 1,162)$, Alaska $(\$ 1,091)$, Louisiana $(\$ 1,041)$, and Oklahoma $(\$ 1,040)$; states with the lowest lifetime costs per capita were New York (\$491), New Jersey (\$533), California (\$538), Massachusetts (\$550), and Minnesota (\$557).

\section{Unintentional Injuries}

West Virginia had the highest lifetime costs per capita for fatal unintentional injuries ( $\$ 815)$, the highest unintentional injury mortality rate among males (95.2), and the highest unintentional injury mortality rate among persons aged 25-64 years (88.5) (Table 1). Maryland had the lowest lifetime costs per capita for fatal unintentional injuries $(\$ 261)$, the lowest total unintentional injury mortality rate (26.4), the lowest male unintentional injury mortality rate (36.9), and the lowest unintentional injury mortality rate among persons

The $M M W R$ series of publications is published by the Center for Surveillance, Epidemiology, and Laboratory Services, Centers for Disease Control and Prevention (CDC), U.S. Department of Health and Human Services, Atlanta, GA 30329-4027.

Suggested citation: [Author names; first three, then et al., if more than six.] [Report title]. MMWR Morb Mortal Wkly Rep 2017;66:[inclusive page numbers].

\section{Centers for Disease Control and Prevention}

Thomas R. Frieden, MD, MPH, Director

Patricia M. Griffin, MD, Acting Associate Director for Science

Joanne Cono, MD, ScM, Director, Office of Science Quality

Chesley L. Richards, MD, MPH, Deputy Director for Public Health Scientific Services

Michael F. Iademarco, MD, MPH, Director, Center for Surveillance, Epidemiology, and Laboratory Services

\section{MMWR Editorial and Production Staff (Weekly)}

Sonja A. Rasmussen, MD, MS, Editor-in-Chief

Charlotte K. Kent, PhD, MPH, Executive Editor Jacqueline Gindler, MD, Editor

Teresa F. Rutledge, Managing Editor

Douglas W. Weatherwax, Lead Technical Writer-Editor

Stacy A. Benton, Soumya Dunworth, PhD, Teresa M. Hood, MS, Technical Writer-Editors

MMWR Editorial Board

Timothy F. Jones, MD, Chairman

Matthew L. Boulton, MD, MPH Virginia A. Caine, MD

Katherine Lyon Daniel, PhD

Jonathan E. Fielding, MD, MPH, MBA

David W. Fleming, MD

\author{
William E. Halperin, MD, DrPH, MPH \\ King K. Holmes, MD, PhD \\ Robin Ikeda, MD, MPH \\ Rima F. Khabbaz, MD \\ Phyllis Meadows, PhD, MSN, RN \\ Jewel Mullen, MD, MPH, MPA
}

Martha F. Boyd, Lead Visual Information Specialist

Maureen A. Leahy, Julia C. Martinroe, Stephen R. Spriggs, Tong Yang, Visual Information Specialists

Quang M. Doan, MBA, Phyllis H. King,

Terraye M. Starr, Moua Yang

Information Technology Specialists

\author{
Jeff Niederdeppe, PhD \\ Patricia Quinlisk, MD, MPH \\ Patrick L. Remington, MD, MPH \\ Carlos Roig, MS, MA \\ William L. Roper, MD, MPH \\ William Schaffner, MD
}


aged 25-64 years (23.3). New Mexico had the highest total unintentional injury mortality rate (71.9) and the highest female unintentional injury mortality rate (49.9). California had the highest lifetime costs for fatal unintentional injuries ( $\$ 12.2$ billion) and the highest number of unintentional injury deaths $(11,804)$.

\section{Suicides}

Alaska and New Jersey had the highest and lowest lifetime suicide costs per capita ( $\$ 338$ and $\$ 107$, respectively) (Table 2). Montana had the highest total suicide rate (23.8), the highest male suicide rate (36.8), and the highest female suicide rate (11.4). DC had the lowest number of suicides (52), total suicide rate (7.7), male suicide rate (12.3), and lifetime costs ( $\$ 73$ million). California had the highest lifetime costs ( $\$ 4.9$ billion) and the highest number of suicides $(4,214)$.

\section{Homicides}

The highest and lowest lifetime homicide-related mortality costs per capita were in DC (\$273) and Hawaii (\$24), respectively (Table 2$).{ }^{\dagger} \mathrm{DC}$ had the highest total homicide rate (13.2), the highest male homicide rate (22.3), and the highest female homicide rate (4.8). New Hampshire, Maine, and Massachusetts had the lowest total homicide rate (1.3), the lowest male homicide rate (2.6), and the lowest female homicide rate $(0.5)$, respectively. California had the highest lifetime homicide-related costs ( $\$ 3.1$ billion) and the highest number of homicides $(1,813)$.

\section{Discussion}

Economic burdens of fatal injuries varied widely in the 50 states and DC for each of the four categories of intent. Across all the four fatal injury intents, some states consistently had lower lifetime costs per capita than most other states. For example, New York, New Jersey, and California ranked among the five lowest states in terms of lifetime costs per capita for injuries of all intents, unintentional injuries, and suicides. In contrast, New Mexico ranked among the five highest states in terms of lifetime costs per capita for injuries of all intents, unintentional injuries, and suicides. Varying economic burdens of fatal injuries in the 50 states and DC might be attributed to the different injury mortality rates, the different medical costs resulting from different medical procedures, and the different

\footnotetext{
$\dagger$ Lifetime costs or lifetime costs per capita of homicides were not presented for New Hampshire, North Dakota, South Dakota, Vermont, and Wyoming because those states had unstable average medical and work loss costs or unstable homicide rates.
}

FIGURE. Costs per capita* of fatal injuries of all intents - United States, 2014

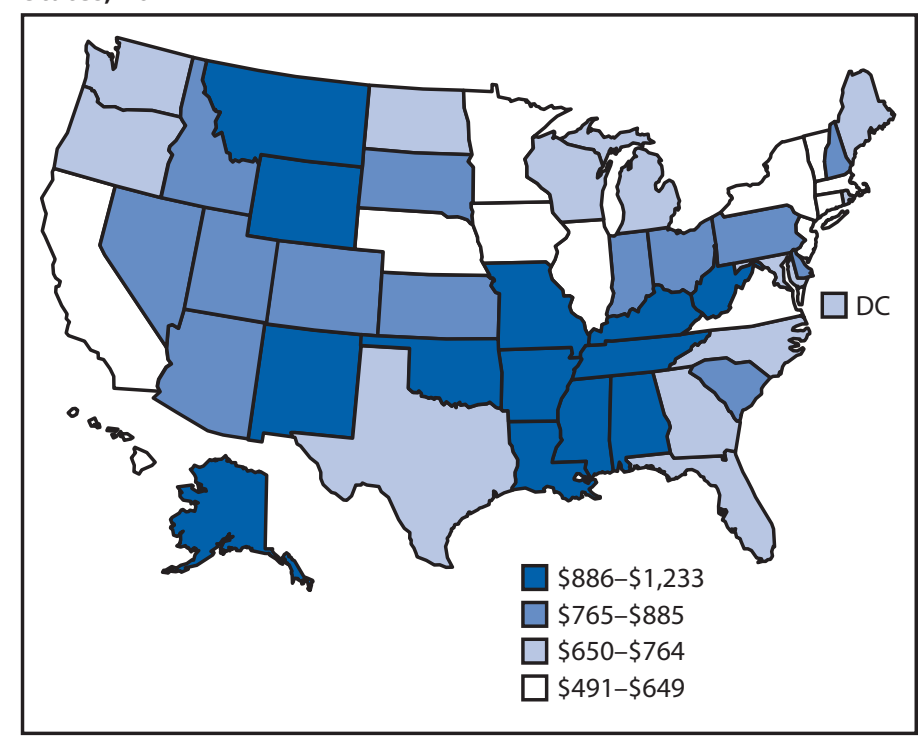

* In 2014 U.S. dollars.

demographic characteristics of injury decedents, such as sex and age.

Implementation of effective injury prevention strategies is needed to help reduce the substantial lifetime medical and work-loss costs associated with fatal injuries. The differing state-level lifetime costs per capita for fatal injuries suggests an urgent need in some states to prevent injuries. States that consistently have lower lifetime costs per capita across different intents of injuries might have successful injury prevention experiences that could be shared with states with higher per capita costs.

The findings in this report are subject to at least four limitations. First, the costs account for medical and work-loss costs associated with decedents. Other societal costs, such as criminal justice costs and the pain and suffering of family members, were not considered. Second, work-loss costs, based on the mean earnings of the general population by sex and specific age groups, might be over- or underestimated because the mean earnings of decedents might differ from those of the general population. Third, intent of fatal injury, as determined from the manner of death assigned on death certificates by coroners or medical examiners, might differ across jurisdictions (5). Finally, unintentional fatal injuries were not broken down into more specific categories such as motor vehicle crashes, drug overdoses, traumatic brain injuries, and older adult falls, so that this report cannot indicate the economic burdens of those specific categories of unintentional injuries.

During 2005-2014, the number of unintentional fatal injuries increased $15 \%$, from 117,809 to 136,053 , and 


\section{Summary}

What is already known about this topic?

Injuries are a leading cause of death in the United States. Injury-associated deaths result in a substantial economic burden to the United States: the total estimated lifetime medical and work-loss costs were $\$ 214$ billion in 2013. Injury and violence prevention strategies can save lives and reduce costs.

What is added by this report?

Lifetime costs and lifetime costs per capita were calculated for each of the 50 states and the District of Columbia (DC) and for each of four injury intent categories (all intents, unintentional, suicide, and homicide) for 2014. Economic burdens varied widely among the states and DC. Lifetime costs per capita ranged from $\$ 1,233$ (New Mexico) to $\$ 491$ (New York) among fatal injuries of all intents, from $\$ 815$ (West Virginia) to $\$ 261$ (Maryland) among unintentional injuries, from $\$ 338$ (Alaska) to \$107 (New Jersey) among suicides, and from \$273 (DC) to \$24 (Hawaii) for homicides.

What are the implications for public health practice?

States can engage more effectively and efficiently in injury prevention if they are aware of the economic burden of injuries, identify areas for immediate improvement, and devote necessary resources to those areas. States that consistently have lower lifetime costs per capita across different intents of injuries might have successful injury prevention experiences that could be shared with states with higher per capita costs.

unintentional injury moved from the fifth to the fourth leading cause of death; the number of suicides rose $31 \%$, from 32,637 to 42,773 , and suicide moved from the eleventh to the tenth leading cause of death $(2,6)$. The increasing incidence and economic burden of injuries, particularly unintentional injuries and suicides, call for effective prevention programs and strategies. For example, the CDC Guideline for Prescribing Opioids for Chronic Pain provides prescribing recommendations for opioid pain medication to patients aged $\geq 18$ years with chronic pain in primary care settings ( 7$)$, which could be adopted by states and might reduce the number of persons who overdose prescribed opioid medications. To reduce motor vehicle crash fatalities, states could increase seatbelt use with primary enforcement seatbelt laws that cover everyone in the vehicle (8) or consider requiring car seats and booster seats for children through at least age 8 years or until seatbelts fit properly (9). The 2012 Surgeon General's National Strategy for Suicide Prevention suggests that strategies enhancing social support, community connectedness, and access to mental health and preventive services and measures to reduce stigma and barriers associated with seeking help might alleviate suicide risk across the lifespan (10). The estimates of state-level economic burdens of fatal injuries will permit policy makers to compare the costs of implementing prevention programs and strategies with the cost savings garnered from the aversion of fatal injuries.

${ }^{1}$ Division of Analysis, Research and Practice Integration, National Center for Injury Prevention and Control, CDC.

Corresponding author: Feijun Luo, fluo@cdc.gov, 770-488-3896.

\section{References}

1. Florence C, Simon T, Haegerich T, Luo F, Zhou C. Estimated lifetime medical and work-loss costs of fatal injuries-United States, 2013. MMWR Morb Mortal Wkly Rep 2015;64:1074-7. http://dx.doi. org/10.15585/mmwr.mm6438a4

2. Kochanek KD, Murphy SL, Xu JQ, Tejada-Vera B. Deaths: final data for 2014. National Vital Statistics Reports, Vol. 65, No. 4. Hyattsville, MD: US Department of Health and Human Services, CDC, National Center for Health Statistics; 2016. https://www.cdc.gov/nchs/data/nvsr/ nvsr65/nvsr65_04.pdf

3. CDC. Web-Based Injury Statistics Query and Reporting System (WISQARS). Atlanta, GA: US Department of Health and Human Services; 2016. https://www.cdc.gov/injury/wisqars

4. Lawrence BA, Miller TA. Medical and work loss cost estimation methods for the WISQARS cost of injury module. Calverton, MD: Pacific Institute for Research \& Evaluation; 2014. http://www.pire.org/ documents/WisqarsCostMethods.pdf

5. Breiding MJ, Wiersema B. Variability of undetermined manner of death classification in the US. Inj Prev 2006;12(Suppl 2):ii49-54. http:// dx.doi.org/10.1136/ip.2006.012591

6. Kung HC, Hoyert DL, Xu JQ, Murphy SL. Deaths: final data for 2005. National Vital Statistics Reports, vol. 52, no. 10. Hyattsville, MD: US Department of Health and Human Services, CDC, National Center for Health Statistics; 2008. https:/www.cdc.gov/nchs/data/nvsr/nvsr56/ nvsr56_10.pdf

7. Dowell D, Haegerich TM, Chou R. CDC guideline for prescribing opioids for chronic pain-United States, 2016. MMWR Recomm Rep 2016;65(No. RR-1). http://dx.doi.org/10.15585/mmwr.rr6501e1

8. CDC. Prevention status reports: motor vehicle injuries. Atlanta, GA: US Department of Health and Human Services, CDC; 2016. https:// wwwn.cdc.gov/psr/NationalSummary/NSMVI.aspx

9. CDC. Buckle up: restraint use state fact sheets. Atlanta, GA: US Department of Health and Human Services, CDC; 2016. https://www. cdc.gov/motorvehiclesafety/seatbelts/states.html

10. Office of the Surgeon General. National Action Alliance for Suicide Prevention. 2012 national strategy for suicide prevention: goals and objectives for action. Washington, DC: US Department of Health and Human Services, Office of the Surgeon General; 2012. https://www. surgeongeneral.gov/library/reports/national-strategy-suicide-prevention/ full-report.pdf 
TABLE 1. Deaths from injuries of all intents and unintentional injuries, rates per 100,000 population, lifetime medical and work-loss costs, and lifetime medical and work-loss costs per capita, by state — United States, 2014

\begin{tabular}{|c|c|c|c|c|c|c|c|c|c|c|c|c|}
\hline \multirow[b]{3}{*}{ State } & \multicolumn{6}{|c|}{ All intents } & \multicolumn{6}{|c|}{ Unintentional injuries } \\
\hline & \multirow[b]{2}{*}{ Total } & \multicolumn{2}{|c|}{ Sex } & \multicolumn{3}{|c|}{ Age group (yrs) } & \multirow[b]{2}{*}{ Total } & \multicolumn{2}{|c|}{ Sex } & \multicolumn{3}{|c|}{ Age group (yrs) } \\
\hline & & Male & Female & $0-24$ & $25-64$ & $\geq 65$ & & Male & Female & $0-24$ & $25-64$ & $\geq 65$ \\
\hline \multicolumn{13}{|l|}{ Alabama } \\
\hline No. of deaths (rate) & $3,625(73.2)$ & $2,440(105.3)$ & $1,185(44.1)$ & 534 (31.6) & 2,224 (89.6) & 867 (121.9) & $2,463(49.2)$ & $1,525(65.9)$ & 938 (34.4) & $360(21.5)$ & $1,396(55.6)$ & 707 (100.5) \\
\hline $\begin{array}{l}\text { Costs, million USD (per } \\
\text { capita, USD*) }\end{array}$ & $4,372(902)$ & 3,317 & 1,038 & 983 & 3,138 & 163 & $2,767(571)$ & 1,967 & 784 & 642 & 1,943 & 130 \\
\hline \multicolumn{13}{|l|}{ Alaska } \\
\hline No. of deaths (rate) & $615(85.8)$ & 441 (119.9) & $174(50.4)$ & $105(36.3)$ & $427(106.1)$ & $83(141.0)$ & $379(54.9)$ & $260(74.2)$ & $119(35.6)$ & $54(18.5)$ & $259(64.7)$ & $66(116.2)$ \\
\hline $\begin{array}{l}\text { Costs, million USD (per } \\
\text { capita, USD*) }\end{array}$ & $804(1,091)$ & 634 & 170 & 193 & 592 & 17 & $448(608)$ & 341 & 109 & $94^{\mathrm{a}}$ & 349 & 13 \\
\hline \multicolumn{13}{|l|}{ Arizona } \\
\hline No. of deaths (rate) & $5,079(72.6)$ & $3,387(100.4)$ & $1,692(45.5)$ & $603(25.1)$ & $2,900(85.4)$ & $1,575(152.7)$ & $3,322(46.8)$ & $2,077(61.5)$ & $1,245(32.5)$ & $357(14.9)$ & $1,710(50.0)$ & $1,254(122.9)$ \\
\hline $\begin{array}{l}\text { Costs, million USD (per } \\
\text { capita, USD*) }\end{array}$ & $5,604(832)$ & 4,326 & 1,259 & 1,129 & 3,942 & 260 & $3,226(479)$ & 2,425 & 816 & 652 & 2,247 & 201 \\
\hline \multicolumn{13}{|l|}{ Arkansas } \\
\hline No. of deaths (rate) & $2,280(75.2)$ & $1,522(105.7)$ & $758(46.4)$ & $316(30.7)$ & 1,330 (89.6) & 634 (140.9) & $1,458(47.2)$ & $907(62.8)$ & $551(32.6)$ & $189(18.4)$ & $757(50.4)$ & $512(114.8)$ \\
\hline $\begin{array}{l}\text { Costs, million USD (per } \\
\text { capita, USD*) }\end{array}$ & $2,719(917)$ & 2,052 & 642 & 587 & 1,904 & 117 & $1,623(547)$ & 1,180 & 420 & 347 & 1,080 & 91 \\
\hline \multicolumn{13}{|l|}{ California } \\
\hline No. of deaths (rate) & $18,152(44.9)$ & $12,820(66.0)$ & $5,332(25.0)$ & $2,495(17.6)$ & $11,109(52.5)$ & $4,544(90.2)$ & $11,804(29.1)$ & $7,847(40.6)$ & $3,957(18.3)$ & $1,437(10.2)$ & $6,832(32.0)$ & $3,531(69.8)$ \\
\hline $\begin{array}{l}\text { Costs, million USD (per } \\
\text { capita, USD*) }\end{array}$ & $20,894(538)$ & 16,746 & 4,209 & 4,760 & 14,766 & 803 & $12,171(314)$ & 9,450 & 2,808 & 2,686 & 8,765 & 601 \\
\hline \multicolumn{13}{|l|}{ Colorado } \\
\hline No. of deaths (rate) & $3,883(72.2)$ & $2,543(98.1)$ & $1,340(46.9)$ & $471(25.3)$ & $2,168(74.5)$ & $1,243(193.4)$ & $2,517(47.1)$ & $1,513(60.1)$ & $1,004(34.4)$ & $254(13.6)$ & $1,221(41.7)$ & $1,042(163.2)$ \\
\hline $\begin{array}{l}\text { Costs, million USD (per } \\
\text { capita, USD*) }\end{array}$ & 4,175 (779) & 3,202 & 989 & 873 & 2,915 & 194 & $2,317(433)$ & 1,691 & 640 & 459 & 1,602 & 154 \\
\hline \multicolumn{13}{|l|}{ Connecticut } \\
\hline No. of deaths (rate) & $2,140(53.9)$ & $1,373(76.7)$ & $767(33.0)$ & 205 (16.6) & $1,152(60.8)$ & $783(129.5)$ & $1,642(40.8)$ & $1,005(56.8)$ & $637(26.4)$ & $120(9.7)$ & $824(44.2)$ & $698(114.1)$ \\
\hline $\begin{array}{l}\text { Costs, million USD (per } \\
\text { capita, USD*) }\end{array}$ & $2,186(608)$ & 1,682 & 464 & 405 & 1,584 & 117 & $1,446(402)$ & 1,100 & 330 & 234 & 1,126 & 100 \\
\hline \multicolumn{13}{|l|}{ Delaware } \\
\hline No. of deaths (rate) & $629(65.8)$ & $433(97.0)$ & $196(37.4)$ & $79(25.0)$ & $383(81.5)$ & $167(114.8)$ & $425(43.9)$ & $270(60.2)$ & $155(29.1)$ & $50(15.8)$ & $239(50.6)$ & $136(94.8)$ \\
\hline $\begin{array}{l}\text { Costs, million USD (per } \\
\text { capita, USD*) }\end{array}$ & 775 (829) & 592 & 184 & 146 & 549 & 31 & $472(505)$ & 334 & 139 & $88^{\dagger}$ & 338 & 24 \\
\hline District of Columbia & & & & & & & & & & & & \\
\hline No. of deaths (rate) & $385(56.2)$ & $264(81.7)$ & $121(33.7)$ & $50(19.8)$ & $250(67.6)$ & $85(111.1)$ & $217(32.7)$ & $130(42.4)$ & $87(24.0)$ & $12\left(4.8^{\S}\right)$ & $133(36.8)$ & $72(94.0)$ \\
\hline $\begin{array}{l}\text { Costs, million USD (per } \\
\text { capita, USD*) }\end{array}$ & $479(726)$ & 370 & 97 & 97 & 357 & 13 & $194(294)$ & 130 & 61 & - & 163 & 11 \\
\hline Florida & & & & & & & & & & & & \\
\hline No. of deaths (rate) & $13,673(61.5)$ & $9,216(88.4)$ & $4,457(35.8)$ & $1,672(26.7)$ & 7,363 (71) & 4,636 (119.5) & $9,433(41.2)$ & $5,932(56.2)$ & $3,501(27)$ & $1,025(16.5)$ & $4,610(44.3)$ & $3,796(97.5)$ \\
\hline $\begin{array}{l}\text { Costs, million USD (per } \\
\text { capita, USD*) }\end{array}$ & $14,763(742)$ & 11,411 & 3,326 & 3,111 & 9,992 & 773 & $9,478(476)$ & 7,055 & 2,386 & 1,859 & 6,301 & 608 \\
\hline Georgia & & & & & & & & & & & & \\
\hline No. of deaths (rate) & $6,002(60.1)$ & 4,061 (85.8) & $1,941(36.5)$ & $946(25.8)$ & $3,589(66.8)$ & $1,467(128.1)$ & $3,964(40.1)$ & $2,491(53.8)$ & $1,473(27.6)$ & $561(15.4)$ & $2,197(40.6)$ & 1,206 (106.9) \\
\hline $\begin{array}{l}\text { Costs, million USD (per } \\
\text { capita, USD*) }\end{array}$ & 7,055 (699) & 5,452 & 1,582 & 1,755 & 4,910 & 271 & 4,232 (419) & 3,117 & 1,104 & 1,009 & 2,927 & 214 \\
\hline Hawaii & & & & & & & & & & & & \\
\hline No. of deaths (rate) & $733(47.3)$ & $527(70.6)$ & $206(24.0)$ & $79(16.1)$ & $428(57.5)$ & $226(92.5)$ & $476(29.9)$ & $327(43.9)$ & $149(16.3)$ & $47(9.6)$ & $246(32.9)$ & $183(73.8)$ \\
\hline $\begin{array}{l}\text { Costs, million USD (per } \\
\text { capita, USD*) }\end{array}$ & $825(581)$ & 684 & 148 & 149 & 588 & 39 & $482(340)$ & 389 & 86 & 86 & 340 & 30 \\
\hline Idaho & & & & & & & & & & & & \\
\hline No. of deaths (rate) & $1,156(71.2)$ & 742 (95.2) & $414(48.4)$ & $172(29.4)$ & $607(75.0)$ & 377 (172.7) & $765(46.5)$ & $457(58.8)$ & $308(35.0)$ & $100(17.1)$ & 341 (41.6) & 324 (149.2) \\
\hline $\begin{array}{l}\text { Costs, million USD (per } \\
\text { capita, USD*) }\end{array}$ & $1,274(780)$ & 916 & 350 & 311 & 814 & 63 & $785(480)$ & 547 & 238 & 177 & 458 & 52 \\
\hline Illinois & & & & & & & & & & & & \\
\hline No. of deaths (rate) & $6,983(52.0)$ & $4,808(75.9)$ & $2,175(29.6)$ & $1,123(25.0)$ & 4,006 (58.3) & 1,853 (101.7) & $4,644(34.2)$ & $2,918(46.5)$ & $1,726(22.9)$ & $557(12.4)$ & $2,506(36.3)$ & $1,581(86.5)$ \\
\hline $\begin{array}{l}\text { Costs, million USD (per } \\
\text { capita, USD*) }\end{array}$ & $8,297(644)$ & 6,550 & 1,697 & 2,104 & 5,759 & 311 & $4,833(375)$ & 3,579 & 1,206 & 1,015 & 3,502 & 256 \\
\hline Indiana & & & & & & & & & & & & \\
\hline No. of deaths (rate) & $4,462(66.5)$ & $3,007(94.1)$ & $1,455(40.1)$ & $687(29.0)$ & 2,685 (79.9) & 1,088 (115.6) & $2,974(43.8)$ & $1,853(58.3)$ & $1,121(30.0)$ & 390 (16.6) & $1,665(49.2)$ & $919(97.3)$ \\
\hline $\begin{array}{l}\text { Costs, million USD (per } \\
\text { capita, USD*) }\end{array}$ & $5,240(794)$ & 4,059 & 1,190 & 1,288 & 3,718 & 196 & $3,166(480)$ & 2,339 & 823 & 716 & 2,279 & 160 \\
\hline lowa & & & & & & & & & & & & \\
\hline No. of deaths (rate) & $2,045(58.4)$ & $1,300(81.2)$ & 745 (37.4) & $237(21.5)$ & $936(58.4)$ & $872(161.7)$ & 1,517 (41.9) & $898(55.8)$ & $619(29.3)$ & $135(12.4)$ & $586(36.3)$ & 796 (146.9) \\
\hline $\begin{array}{l}\text { Costs, million USD (per } \\
\text { capita, USD*) }\end{array}$ & $1,987(639)$ & 1,493 & 479 & 435 & 1,242 & 140 & 1,292 (416) & 937 & 343 & 243 & 756 & 123 \\
\hline
\end{tabular}

See table footnotes on page 8. 
TABLE 1. (Continued) Deaths from injuries of all intents and unintentional injuries, rates per 100,000 population, lifetime medical and work-loss costs, and lifetime medical and work-loss costs per capita, by state — United States, 2014

\begin{tabular}{|c|c|c|c|c|c|c|c|c|c|c|c|c|}
\hline \multirow[b]{3}{*}{ State } & \multicolumn{6}{|c|}{ All intents } & \multicolumn{6}{|c|}{ Unintentional injuries } \\
\hline & \multirow[b]{2}{*}{ Total } & \multicolumn{2}{|c|}{ Sex } & \multicolumn{3}{|c|}{ Age group (yrs) } & \multirow[b]{2}{*}{ Total } & \multicolumn{2}{|c|}{ Sex } & \multicolumn{3}{|c|}{ Age group (yrs) } \\
\hline & & Male & Female & $0-24$ & $25-64$ & $\geq 65$ & & Male & Female & $0-24$ & $25-64$ & $\geq 65$ \\
\hline \multicolumn{13}{|l|}{ Kansas } \\
\hline No. of deaths (rate) & $1,987(65.2)$ & $1,292(89.8)$ & $695(41.7)$ & $266(24.7)$ & $1,046(71.2)$ & $675(154)$ & $1,377(44.1)$ & $829(57.4)$ & $548(31.5)$ & $157(14.6)$ & $634(42.7)$ & $586(132.3)$ \\
\hline $\begin{array}{l}\text { Costs, million USD (per } \\
\text { capita, USD*) }\end{array}$ & $2,223(765)$ & 1,697 & 505 & 491 & 1,438 & 115 & $1,367(471)$ & 1,004 & 339 & 284 & 855 & 97 \\
\hline \multicolumn{13}{|l|}{ Kentucky } \\
\hline No. of deaths (rate) & $3,634(80.7)$ & $2,466(114.5)$ & $1,168(48.8)$ & $427(27.8)$ & $2,343(102.7)$ & $864(138.4)$ & $2,622(58.3)$ & $1,677(78.8)$ & $945(39.1)$ & $296(19.3)$ & $1,616(71)$ & $710(114.9)$ \\
\hline $\begin{array}{l}\text { Costs, million USD (per } \\
\text { capita, USD*) }\end{array}$ & $4,296(973)$ & 3,300 & 1,010 & 767 & 3,314 & 164 & $2,966(672)$ & 2,196 & 775 & 523 & 2,293 & 131 \\
\hline \multicolumn{13}{|l|}{ Louisiana } \\
\hline No. of deaths (rate) & $3,654(77.5)$ & $2,576(113.8)$ & $1,078(43.7)$ & 659 (39.6) & $2,334(95.9)$ & $659(107.8)$ & $2,344(49.6)$ & $1,584(70.5)$ & $760(30.4)$ & $381(22.9)$ & $1,440(58.9)$ & $522(86.1)$ \\
\hline $\begin{array}{l}\text { Costs, million USD (per } \\
\text { capita, USD*) }\end{array}$ & $4,839(1,041)$ & 3,805 & 1,008 & 1,233 & 3,430 & 127 & $2,855(614)$ & 2,203 & 666 & 685 & 2,045 & 97 \\
\hline \multicolumn{13}{|l|}{ Maine } \\
\hline No. of deaths (rate) & $952(65.0)$ & $633(93.4)$ & $319(38.5)$ & $105(26.5)$ & $492(71.3)$ & 354 (146.8) & 690 (45.9) & $429(63.1)$ & $261(30.0)$ & $63(15.9)$ & $320(46.7)$ & $306(126.7)$ \\
\hline $\begin{array}{l}\text { Costs, million USD (per } \\
\text { capita, USD*) }\end{array}$ & $960(722)$ & 736 & 215 & $200^{a}$ & 649 & 60 & $626(470)$ & 467 & 152 & $117^{\dagger}$ & 423 & 50 \\
\hline \multicolumn{13}{|l|}{ Maryland } \\
\hline No. of deaths (rate) & $3,482(56.1)$ & $2,426(83.7)$ & $1,056(31.0)$ & $462(22.6)$ & $2,129(65.9)$ & 891 (109.3) & $1,674(26.4)$ & $1,046(36.9)$ & $628(17.3)$ & $183(9)$ & $772(23.3)$ & $719(88.3)$ \\
\hline $\begin{array}{l}\text { Costs, million USD (per } \\
\text { capita, USD*) }\end{array}$ & $4,233(708)$ & 3,376 & 838 & 888 & 3,049 & 149 & $1,560(261)$ & 1,183 & 363 & 340 & 1,039 & 114 \\
\hline \multicolumn{13}{|l|}{ Massachusetts } \\
\hline No. of deaths (rate) & $3,452(47.4)$ & 2,361 (70.4) & $1,091(26.4)$ & $335(13.8)$ & $2,132(59.4)$ & $984(92.1)$ & $2,692(36.8)$ & $1,767(53.2)$ & $925(21.9)$ & $230(9.4)$ & $1,577(44.3)$ & $884(82.4)$ \\
\hline $\begin{array}{l}\text { Costs, million USD (per } \\
\text { capita, USD*) }\end{array}$ & $3,707(550)$ & 3,032 & 711 & 648 & 2,936 & 158 & $2,508(372)$ & 2,059 & 503 & 444 & 2,143 & 138 \\
\hline \multicolumn{13}{|l|}{ Michigan } \\
\hline No. of deaths (rate) & $6,652(63.8)$ & 4,392 (89.2) & $2,260(39.9)$ & $967(27.6)$ & $3,807(74.2)$ & $1,878(122.5)$ & $4,422(41.5)$ & $2,714(55.0)$ & $1,708(28.9)$ & $532(15.4)$ & $2,283(43.9)$ & $1,607(104.6)$ \\
\hline $\begin{array}{l}\text { Costs, million USD (per } \\
\text { capita, USD*) }\end{array}$ & $7,539(761)$ & 5,766 & 1,749 & 1,780 & 5,194 & 322 & $4,338(438)$ & 3,168 & 1,172 & 943 & 3,014 & 264 \\
\hline \multicolumn{13}{|l|}{ Minnesota } \\
\hline No. of deaths (rate) & $3,226(54.3)$ & $1,956(71.6)$ & $1,270(37.4)$ & $361(19.6)$ & $1,465(50.0)$ & $1,400(168.8)$ & 2,385 (39.2) & $1,327(49.0)$ & $1,058(29.8)$ & $197(10.7)$ & $888(30.3)$ & $1,300(155.9)$ \\
\hline $\begin{array}{l}\text { Costs, million USD (per } \\
\text { capita, USD*) }\end{array}$ & $3,041(557)$ & 2,227 & 771 & 670 & 1,953 & 210 & $1,855(340)$ & 1,296 & 526 & 358 & 1,137 & 190 \\
\hline Mississippi & & & & & & & & & & & & \\
\hline No. of deaths (rate) & $2,477(81.8)$ & $1,702(120.0)$ & $775(47.4)$ & $443(40.7)$ & $1,421(93.4)$ & $612(149)$ & $1,712(56.2)$ & $1,085(77.0)$ & $627(37.8)$ & $288(26.9)$ & $903(58.7)$ & $520(127.4)$ \\
\hline $\begin{array}{l}\text { Costs, million USD (per } \\
\text { capita, USD*) }\end{array}$ & 2,872 (959) & 2,306 & 601 & 807 & 1,963 & 110 & $1,816(607)$ & 1,379 & 447 & 512 & 1,215 & 92 \\
\hline Missouri & & & & & & & & & & & & \\
\hline No. of deaths (rate) & $4,672(74.1)$ & $3,142(105.9)$ & $1,530(43.9)$ & $675(32.2)$ & $2,658(85.7)$ & 1,339 (143.6) & $3,110(48.5)$ & $1,911(64.3)$ & $1,199(33.4)$ & 407 (19.6) & $1,585(50.9)$ & $1,118(119.7)$ \\
\hline $\begin{array}{l}\text { Costs, million USD (per } \\
\text { capita, USD*) }\end{array}$ & $5,371(886)$ & 4,213 & 1,159 & 1,249 & 3,767 & 230 & $3,203(528)$ & 2,379 & 830 & 731 & 2,185 & 186 \\
\hline Montana & & & & & & & & & & & & \\
\hline No. of deaths (rate) & $902(83.1)$ & $586(110.5)$ & $316(56.2)$ & $121(34.9)$ & $475(91.4)$ & 306 (183.8) & $581(52.6)$ & $343(64.8)$ & $238(40.5)$ & $75(21.8)$ & $253(49.1)$ & $253(153.3)$ \\
\hline $\begin{array}{l}\text { Costs, million USD (per } \\
\text { capita, USD*) }\end{array}$ & $973(950)$ & 729 & 239 & 219 & 653 & 55 & $579(566)$ & 411 & 160 & 133 & 359 & 44 \\
\hline Nebraska & & & & & & & & & & & & \\
\hline No. of deaths (rate) & $1,116(56.0)$ & $752(80.6)$ & $364(32.9)$ & $161(23.7)$ & $563(58.9)$ & 392 (134.7) & $781(38.2)$ & $492(52.8)$ & $289(24.7)$ & 95 (13.9) & $338(35.1)$ & $348(118.5)$ \\
\hline $\begin{array}{l}\text { Costs, million USD (per } \\
\text { capita, USD*) }\end{array}$ & $1,139(605)$ & 913 & 245 & 296 & 754 & 61 & $697(370)$ & 543 & 169 & 172 & 446 & 52 \\
\hline Nevada & & & & & & & & & & & & \\
\hline No. of deaths (rate) & $1,948(67.0)$ & $1,359(94.6)$ & 589 (39.7) & $251(26.6)$ & $1251(81.2)$ & 446 (121.7) & $1,166(40.1)$ & $750(52.1)$ & $416(28.1)$ & $144(15.3)$ & $722(46.4)$ & $300(83.6)$ \\
\hline $\begin{array}{l}\text { Costs, million USD (per } \\
\text { capita, USD*) }\end{array}$ & $2,294(808)$ & 1,781 & 534 & 464 & 1,665 & 85 & $1,319(465)$ & 975 & 359 & 265 & 949 & 54 \\
\hline New Hampshire & & & & & & & & & & & & \\
\hline No. of deaths (rate) & $1,001(70.8)$ & 645 (97.9) & $356(44.6)$ & $92(20.1)$ & $584(84.9)$ & 325 (154.8) & $716(50.4)$ & $435(67.2)$ & $281(34.2)$ & $56(12.1)$ & $376(55.7)$ & 284 (135.6) \\
\hline $\begin{array}{l}\text { Costs, million USD (per } \\
\text { capita, USD*) }\end{array}$ & $1,022(771)$ & 800 & 226 & 174 & 798 & 55 & $664(500)$ & 515 & 156 & 104 & 522 & 46 \\
\hline New Jersey & & & & & & & & & & & & \\
\hline No. of deaths (rate) & $4,210(44.4)$ & $2,881(65.2)$ & $1,329(25.4)$ & $555(18.8)$ & $2,454(51.1)$ & $1,200(88.4)$ & $2,970(30.8)$ & $1,935(43.9)$ & $1,035(19.1)$ & $319(10.8)$ & $1,597(33.1)$ & $1,053(77.2)$ \\
\hline $\begin{array}{l}\text { Costs, million USD (per } \\
\text { capita, USD*) }\end{array}$ & $4,765(533)$ & 3,806 & 961 & 1,074 & 3,465 & 201 & $2,991(335)$ & 2,368 & 657 & 607 & 2,238 & 171 \\
\hline New Mexico & & & & & & & & & & & & \\
\hline No. of deaths (rate) & 2,163 (101.9) & $1,443(141.1)$ & $720(63.7)$ & $291(38.5)$ & $1,303(124.7)$ & 569 (185.9) & 1,534 (71.9) & $958(94.3)$ & $576(49.9)$ & $173(22.8)$ & 899 (85.7) & $462(152.4)$ \\
\hline $\begin{array}{l}\text { Costs, million USD (per } \\
\text { capita, USD*) }\end{array}$ & $2,573(1,233)$ & 1,965 & 603 & 542 & 1,844 & 101 & $1,659(796)$ & 1,214 & 445 & 315 & 1,250 & 79 \\
\hline
\end{tabular}

See table footnotes on page 8 . 
TABLE 1. (Continued) Deaths from injuries of all intents and unintentional injuries, rates per 100,000 population, lifetime medical and work-loss costs, and lifetime medical and work-loss costs per capita, by state - United States, 2014

\begin{tabular}{|c|c|c|c|c|c|c|c|c|c|c|c|c|}
\hline \multirow[b]{3}{*}{ State } & \multicolumn{6}{|c|}{ All intents } & \multicolumn{6}{|c|}{ Unintentional injuries } \\
\hline & \multirow[b]{2}{*}{ Total } & \multicolumn{2}{|c|}{ Sex } & \multicolumn{3}{|c|}{ Age group (yrs) } & \multirow[b]{2}{*}{ Total } & \multicolumn{2}{|c|}{ Sex } & \multicolumn{3}{|c|}{ Age group (yrs) } \\
\hline & & Male & Female & $0-24$ & $25-64$ & $\geq 65$ & & Male & Female & $0-24$ & $25-64$ & $\geq 65$ \\
\hline \multicolumn{13}{|l|}{ New York } \\
\hline No. of deaths (rate) & $8,585(40.2)$ & $5,801(58.9)$ & $2,784(23.1)$ & 1,046 & 4,934 (45.9) & $2,600(87.1)$ & $5,945(27.5)$ & $3,799(38.8)$ & 2,146 & $587(8.5)$ & $3,095(28.7)$ & $2,259(75.5)$ \\
\hline $\begin{array}{l}\text { Costs, million USD (per } \\
\text { capita, USD*) }\end{array}$ & $9,689(491)$ & 7,594 & 1,987 & 1,987 & 6,858 & 436 & $5,772(292)$ & 4,443 & 1,302 & 1,095 & 4,158 & 363 \\
\hline \multicolumn{13}{|l|}{ North Carolina } \\
\hline No. of deaths (rate) & $6,541(63.7)$ & $4,358(90.8)$ & $2,183(39.2)$ & $890(25.6)$ & $3,709(71.0)$ & $1,940(140.2)$ & $4,558(44.3)$ & $2,881(60.9)$ & 1,677 & $552(16.0)$ & $2,378(45.4)$ & $1,626(118.7)$ \\
\hline $\begin{array}{l}\text { Costs, million USD (per } \\
\text { capita, USD*) }\end{array}$ & $7,310(735)$ & 5,674 & 1,607 & 1,681 & 5,148 & 334 & $4,620(465)$ & 3,517 & 1,093 & 1,021 & 3,255 & 270 \\
\hline \multicolumn{13}{|l|}{ North Dakota } \\
\hline No. of deaths (rate) & $514(64.1)$ & $353(89.5)$ & $161(38.6)$ & $82(27.0)$ & $258(68.6)$ & $174(149.1)$ & $349(42.8)$ & $219(56.8)$ & $130(29.4)$ & $44(14.6)$ & $146(39.3)$ & $159(135.8)$ \\
\hline $\begin{array}{l}\text { Costs, million USD (per } \\
\text { capita, USD*) }\end{array}$ & $545(737)$ & 447 & 100 & $158^{\dagger}$ & 367 & 30 & $312(422)$ & 245 & 69 & $82^{\dagger}$ & 205 & 26 \\
\hline \multicolumn{13}{|l|}{ Ohio } \\
\hline No. of deaths (rate) & $8,366(69.4)$ & $5,541(97.9)$ & $2,825(42.9)$ & $984(24.8)$ & $5,062(85.5)$ & $2,320(128.0)$ & $6,178(50.6)$ & $3,828(68.0)$ & 2,350 & $576(14.5)$ & $3,595(60.6)$ & $2,007(110.6)$ \\
\hline $\begin{array}{l}\text { Costs, million USD (per } \\
\text { capita, USD*) }\end{array}$ & $9,370(808)$ & 7,217 & 2,143 & 1,820 & 7,038 & 403 & $6,200(535)$ & 4,607 & 1,609 & 1,041 & 4,874 & 338 \\
\hline \multicolumn{13}{|l|}{ Oklahoma } \\
\hline No. of deaths (rate) & $3,522(88.8)$ & 2,277 (119.9) & $1,245(59.6)$ & 485 & $2,069(104.3)$ & $968(176.8)$ & $2,421(60.3)$ & $1,465(77.3)$ & $956(44.5)$ & $283(20.3)$ & $1,308(65.0)$ & $830(152.5)$ \\
\hline $\begin{array}{l}\text { Costs, million USD (per } \\
\text { capita, USD*) }\end{array}$ & $4,035(1,040)$ & 3,024 & 981 & 893 & 2,841 & 171 & $2,508(647)$ & 1,812 & 686 & 511 & 1,747 & 141 \\
\hline \multicolumn{13}{|l|}{ Oregon } \\
\hline No. of deaths (rate) & $2,773(64.1)$ & $1,805(88.6)$ & $968(40.8)$ & $286(22.1)$ & $1,477(69.0)$ & $1,010(161.8)$ & $1,803(40.8)$ & $1,072(52.7)$ & $731(29.5)$ & $156(12.1)$ & $826(38.3)$ & $821(131.8)$ \\
\hline $\begin{array}{l}\text { Costs, million USD (per } \\
\text { capita, USD*) }\end{array}$ & $2,704(681)$ & 2,075 & 624 & 530 & 1,932 & 159 & $1,504(379)$ & 1,111 & 383 & 285 & 1,068 & 122 \\
\hline \multicolumn{13}{|l|}{ Pennsylvania } \\
\hline No. of deaths (rate) & $9,224(66.1)$ & $6,111(94.1)$ & $3,113(40.0)$ & 1,102 & $5,245(78.8)$ & $2,875(127.4)$ & $6,640(46.6)$ & $4,091(63.0)$ & 2,549 & $683(15.8)$ & $3,454(52.1)$ & $2,503(109.7)$ \\
\hline $\begin{array}{l}\text { Costs, million USD (per } \\
\text { capita, USD*) }\end{array}$ & $10,089(789)$ & 7,874 & 2,229 & 2,085 & 7,225 & 477 & $6,420(502)$ & 4,820 & 1,633 & 1,256 & 4,687 & 404 \\
\hline \multicolumn{13}{|l|}{ Rhode Island } \\
\hline No. of deaths (rate) & $748(62.8)$ & $475(88.8)$ & $273(40.0)$ & $59(15.2)$ & $422(75.6)$ & 267 (143.4) & $592(49.0)$ & $360(67.9)$ & $232(32.7)$ & $33(8.6)$ & $316(57.0)$ & $243(129.3)$ \\
\hline $\begin{array}{l}\text { Costs, million USD (per } \\
\text { capita, USD*) }\end{array}$ & $771(731)$ & 576 & 179 & 113 & 578 & 41 & $526(498)$ & 387 & 134 & $62^{\dagger}$ & 420 & 36 \\
\hline South Carolina & & & & & & & & & & & & \\
\hline No. of deaths (rate) & $3,608(72.0)$ & $2,422(103.1)$ & $1,186(44.0)$ & 564 & $2,111(83.4)$ & $933(132.2)$ & $2,436(48.2)$ & $1,519(65.0)$ & $917(33.4)$ & $334(20.3)$ & $1,333(52.1)$ & $769(110.0)$ \\
\hline $\begin{array}{l}\text { Costs, million USD (per } \\
\text { capita, USD*) }\end{array}$ & $4,279(885)$ & 3,309 & 962 & 1,054 & 2,925 & 169 & $2,693(557)$ & 1,984 & 695 & 615 & 1,821 & 136 \\
\hline South Dakota & & & & & & & & & & & & \\
\hline No. of deaths (rate) & $642(71.1)$ & $415(97.4)$ & $227(45.9)$ & $110(35.9)$ & $320(75.8)$ & $212(149.9)$ & $462(49.2)$ & $282(65.6)$ & $180(34.3)$ & $67(22.1)$ & $195(45.6)$ & 200 (139.9) \\
\hline $\begin{array}{l}\text { Costs, million USD (per } \\
\text { capita, USD*) }\end{array}$ & $687(805)$ & 505 & 172 & 197 & 448 & 35 & $422(495)$ & 302 & 111 & $119^{\dagger}$ & 270 & 31 \\
\hline Tennessee & & & & & & & & & & & & \\
\hline No. of deaths (rate) & $5,237(77.4)$ & $3,489(110.5)$ & $1,748(47.2)$ & 631 & $3,093(90.2)$ & $1,512(163.1)$ & $3,781(55.5)$ & $2,361(75.3)$ & 1,420 & $361(16.0)$ & $2,116(61.4)$ & $1,304(141.7)$ \\
\hline $\begin{array}{l}\text { Costs, million USD (per } \\
\text { capita, USD*) }\end{array}$ & $5,947(908)$ & 4,556 & 1,396 & 1,162 & 4,262 & 273 & $3,900(595)$ & 2,871 & 1,030 & 650 & 2,843 & 228 \\
\hline Texas & & & & & & & & & & & & \\
\hline No. of deaths (rate) & $14,652(55.6)$ & $10,164(79.8)$ & $4,488(32.8)$ & 2,454 & $8,777(62.2)$ & $3,419(115.9)$ & $9,723(37.2)$ & $6,398(51.2)$ & 3,325 & $1,498(14.9)$ & 5,434 & 2,789 (95.4) \\
\hline $\begin{array}{l}\text { Costs, million USD (per } \\
\text { capita, USD*) }\end{array}$ & $17,522(650)$ & 13,869 & 3,740 & 4,549 & 12,340 & 615 & $10,648(395)$ & 8,237 & 2,512 & 2,720 & 7,485 & 486 \\
\hline Utah & & & & & & & & & & & & \\
\hline No. of deaths (rate) & $1,924(73.0)$ & $1,265(97.1)$ & 659 (49.7) & $286(23.5)$ & $1,190(85.7)$ & 446 (158.9) & $1,167(45.3)$ & $726(57.5)$ & $441(33.5)$ & $141(11.5)$ & $662(47.6)$ & $364(130.0)$ \\
\hline $\begin{array}{l}\text { Costs, million USD (per } \\
\text { capita, USD*) }\end{array}$ & $2,362(803)$ & 1,794 & 564 & 525 & 1,726 & 78 & $1,251(425)$ & 937 & 315 & 250 & 942 & 61 \\
\hline Vermont & & & & & & & & & & & & \\
\hline No. of deaths (rate) & $478(68.2)$ & $291(91.0)$ & $187(45.4)$ & $54(24.6)$ & $208(64.2)$ & $216(207.0)$ & $322(44.4)$ & $168(53.3)$ & 154 (34.9) & 25 (10.9) & $112(34.5)$ & $185(179.2)$ \\
\hline $\begin{array}{l}\text { Costs, million USD (per } \\
\text { capita, USD*) }\end{array}$ & $406(648)$ & 314 & 88 & $102^{\dagger}$ & 265 & 32 & $228(365)$ & 161 & 62 & $46^{\dagger}$ & 140 & 27 \\
\hline Virginia & & & & & & & & & & & & \\
\hline No. of deaths (rate) & $4,701(54.7)$ & $3,141(77.2)$ & $1,560(33.7)$ & 634 & $2,618(57.9)$ & 1,449 (132.9) & $3,147(36.7)$ & $1,962(49.2)$ & 1,185 & $362(12.5)$ & $1,577(34.9)$ & $1,208(111.6)$ \\
\hline $\begin{array}{l}\text { Costs, million USD (per } \\
\text { capita, USD*) }\end{array}$ & $5,166(620)$ & 3,996 & 1,128 & 1,196 & 3,655 & 244 & $3,004(361)$ & 2,265 & 720 & 671 & 2,163 & 194 \\
\hline Washington & & & & & & & & & & & & \\
\hline No. of deaths (rate) & $4,428(59.6)$ & 2,909 (81.9) & $1,519(38.2)$ & $530(22.0)$ & $2,446(63.3)$ & $1,451(149.5)$ & 2,997 (39.9) & $1,821(51.8)$ & 1,176 & 304 (12.6) & $1,451(37.0)$ & $1,242(128.6)$ \\
\hline $\begin{array}{l}\text { Costs, million USD (per } \\
\text { capita, USD*) }\end{array}$ & $4,600(651)$ & 3,550 & 1,052 & 1,004 & 3,262 & 240 & $2,727(386)$ & 2,020 & 708 & 564 & 1,873 & 197 \\
\hline
\end{tabular}

See table footnotes on page 8 . 
TABLE 1. (Continued) Deaths from injuries of all intents and unintentional injuries, rates per 100,000 population, lifetime medical and work-loss costs, and lifetime medical and work-loss costs per capita, by state — United States, 2014

\begin{tabular}{|c|c|c|c|c|c|c|c|c|c|c|c|c|}
\hline \multirow[b]{3}{*}{ State } & \multicolumn{6}{|c|}{ All intents } & \multicolumn{6}{|c|}{ Unintentional injuries } \\
\hline & \multirow[b]{2}{*}{ Total } & \multicolumn{2}{|c|}{ Sex } & \multicolumn{3}{|c|}{ Age group (yrs) } & \multirow[b]{2}{*}{ Total } & \multicolumn{2}{|c|}{ Sex } & \multicolumn{3}{|c|}{ Age group (yrs) } \\
\hline & & Male & Female & $0-24$ & $25-64$ & $\geq 65$ & & Male & Female & $0-24$ & $25-64$ & $\geq 65$ \\
\hline \multicolumn{13}{|l|}{ West Virginia } \\
\hline No. of deaths (rate) & $1,897(98.0)$ & $1,253(134.8)$ & $644(62.6)$ & 201 (33.9) & $1,170(125.0)$ & $526(166.2)$ & $1,380(71.1)$ & $874(95.2)$ & $506(47.9)$ & $122(20.6)$ & $818(88.5)$ & $440(140.5)$ \\
\hline $\begin{array}{l}\text { Costs, million USD (per } \\
\text { capita, USD*) }\end{array}$ & $2,149(1,162)$ & 1,599 & 530 & 369 & 1,618 & 94 & $1,507(815)$ & 1,099 & 393 & 225 & 1,133 & 77 \\
\hline \multicolumn{13}{|l|}{ Wisconsin } \\
\hline No. of deaths (rate) & $4,032(64.2)$ & $2,463(85.0)$ & $1,569(43.7)$ & $480(24.1)$ & $1,965(64.8)$ & $1,587(174.2)$ & $3,015(46.7)$ & $1,696(58.4)$ & $1,319(35.1)$ & $275(13.8)$ & $1,279(41.6)$ & $1,461(159.6)$ \\
\hline $\begin{array}{l}\text { Costs, million USD (per } \\
\text { capita, USD*) }\end{array}$ & $3,934(683)$ & 2,895 & 967 & 906 & 2,617 & 229 & $2,499(434)$ & 1,765 & 700 & 508 & 1,665 & 203 \\
\hline \multicolumn{13}{|l|}{ Wyoming } \\
\hline No. of deaths (rate) & $514(86.6)$ & $355(119.2)$ & $159(52.2)$ & $81(39.6)$ & $322(105.3)$ & $111(141.4)$ & $361(60.2)$ & $234(78.4)$ & $127(40.8)$ & $46(22.3)$ & $225(72.4)$ & $90(116.0)$ \\
\hline $\begin{array}{l}\text { Costs, million USD (per } \\
\text { capita, USD*) }\end{array}$ & $581(995)$ & 454 & 134 & $149^{\dagger}$ & 415 & 21 & 384 (658) & 286 & 103 & $83^{\dagger}$ & 291 & 17 \\
\hline
\end{tabular}

* Costs per capita calculated only for totals.

+ Average medical cost was statistically unstable; however, it accounted for less than $1 \%$ of combined average cost.

$\S$ Rates based on $\leq 20$ deaths might be unstable.

I Both average medical cost and average work loss cost were statistically unstable.

TABLE 2. Suicide and homicide deaths, rates per 100,000 population, lifetime medical and work-loss costs, and lifetime medical and work-loss costs per capita, by state - United States, 2014

\begin{tabular}{|c|c|c|c|c|c|c|}
\hline \multirow[b]{3}{*}{ State } & \multicolumn{3}{|c|}{ Suicides } & \multicolumn{3}{|c|}{ Homicides } \\
\hline & \multirow[b]{2}{*}{ Total } & \multicolumn{2}{|c|}{ Sex } & \multirow[b]{2}{*}{ Total } & \multicolumn{2}{|c|}{ Sex } \\
\hline & & Male & Female & & Male & Female \\
\hline \multicolumn{7}{|l|}{ Alabama } \\
\hline No. deaths (rate) & $715(14.5)$ & $569(24.3)$ & $146(5.6)$ & $374(8.0)$ & $304(13.4)$ & $70(2.8)$ \\
\hline Costs, million USD (per capita, USD*) & $897(185)$ & 755 & 143 & $606(125)$ & 532 & $76^{+}$ \\
\hline \multicolumn{7}{|l|}{ Alaska } \\
\hline No. deaths (rate) & $167(22.0)$ & $138(34.8)$ & $29(7.9)$ & $37(4.7)$ & $22(5.3)$ & $15(4.1)$ \\
\hline Costs, million USD (per capita, USD*) & $249^{\dagger}(338)$ & $220^{+}$ & $32^{\dagger}$ & $61^{\dagger}(83)$ & $38^{\dagger}$ & -** \\
\hline \multicolumn{7}{|l|}{ Arizona } \\
\hline No. deaths (rate) & $1,244(18.0)$ & $945(27.7)$ & $299(8.7)$ & $322(5.0)$ & $249(7.7)$ & $73(2.2)$ \\
\hline Costs, million USD (per capita, USD*) & $1,528(227)$ & 1,222 & 293 & $538(80)$ & 448 & $82^{\dagger}$ \\
\hline \multicolumn{7}{|l|}{ Arkansas } \\
\hline No. deaths (rate) & $515(17.2)$ & $406(27.9)$ & $109(7.2)$ & $217(7.6)$ & $158(11.3)$ & $59(4.1)$ \\
\hline Costs, million USD (per capita, USD*) & $671(226)$ & 550 & $119^{\dagger}$ & $323(109)$ & 258 & $62^{\dagger}$ \\
\hline \multicolumn{7}{|l|}{ California } \\
\hline No. deaths (rate) & $4,214(10.5)$ & $3,234(16.7)$ & $980(4.7)$ & $1,813(4.6)$ & $1,514(7.6)$ & $299(1.5)$ \\
\hline Costs, million USD (per capita, USD*) & $4,927(127)$ & 3,986 & 933 & $3,103(80)$ & 2,794 & 337 \\
\hline \multicolumn{7}{|l|}{ Colorado } \\
\hline No. deaths (rate) & $1,083(19.8)$ & $843(31.3)$ & $240(8.7)$ & $177(3.3)$ & $124(4.5)$ & $53(2.1)$ \\
\hline Costs, million USD (per capita, USD*) & $1,421(265)$ & 1,174 & 252 & $282(53)$ & 215 & $58^{\dagger}$ \\
\hline \multicolumn{7}{|l|}{ Connecticut } \\
\hline No. deaths (rate) & $379(9.7)$ & $276(14.8)$ & $103(5.1)$ & $99(2.8)$ & $75(4.3)$ & $24(1.3)$ \\
\hline Costs, million USD (per capita, USD*) & $475(132)$ & 368 & $98^{\dagger}$ & $170(47)$ & 142 & $25^{+}$ \\
\hline \multicolumn{7}{|l|}{ Delaware } \\
\hline No. deaths (rate) & $126(13.2)$ & $100(22.3)$ & $26(5.3)$ & $57(6.5)$ & $47(10.9)$ & $10(2.2)$ \\
\hline Costs, million USD (per capita, USD*) & $168^{\dagger}(179)$ & $140^{\dagger}$ & - $^{* *}$ & $98(105)$ & $87^{\dagger}$ & -** \\
\hline \multicolumn{7}{|l|}{ District of Columbia } \\
\hline No. deaths (rate) & $52(7.7)$ & $39(12.3)$ & $13(4.0)$ & $97(13.2)$ & $79(22.3)$ & $18(4.8)$ \\
\hline Costs, million USD (per capita, USD*) & $73^{\dagger}(110)$ & $59^{\dagger}$ & - $^{* *}$ & $180(273)$ & 152 & - ${ }^{* *}$ \\
\hline \multicolumn{7}{|l|}{ Florida } \\
\hline No. deaths (rate) & $3,035(13.8)$ & $2,328(21.9)$ & $707(6.3)$ & $1,158(6.2)$ & $915(9.8)$ & $243(2.5)$ \\
\hline Costs, million USD (per capita, USD*) & $3,332(167)$ & 2,701 & 624 & $1,852(93)$ & 1,584 & 282 \\
\hline \multicolumn{7}{|l|}{ Georgia } \\
\hline No. deaths (rate) & $1,294(12.6)$ & $998(20.6)$ & $296(5.6)$ & $658(6.5)$ & $518(10.2)$ & $140(2.8)$ \\
\hline Costs, million USD (per capita, USD*) & $1,622(161)$ & 1,323 & 292 & $1,087(108)$ & 933 & 155 \\
\hline
\end{tabular}

See table footnotes on page 11. 
TABLE 2. (Continued) Suicide and homicide deaths, rates per 100,000 population, lifetime medical and work-loss costs, and lifetime medical and work-loss costs per capita, by state — United States, 2014

\begin{tabular}{|c|c|c|c|c|c|c|}
\hline \multirow[b]{3}{*}{ State } & \multicolumn{3}{|c|}{ Suicides } & \multicolumn{3}{|c|}{ Homicides } \\
\hline & \multirow[b]{2}{*}{ Total } & \multicolumn{2}{|c|}{ Sex } & \multirow[b]{2}{*}{ Total } & \multicolumn{2}{|c|}{ Sex } \\
\hline & & Male & Female & & Male & Female \\
\hline \multicolumn{7}{|l|}{ Hawaii } \\
\hline No. deaths (rate) & $204(13.6)$ & $163(21.5)$ & $41(5.4)$ & $30(2.3)$ & $21(3.0)$ & †† \\
\hline \multicolumn{6}{|l|}{ Idaho } & -** \\
\hline No. deaths (rate) & $320(20.1)$ & $240(30.5)$ & $80(10.1)$ & $36(2.4)$ & $22(3.0)$ & $14(1.7)$ \\
\hline Costs, million USD (per capita, USD*) & $391^{\dagger}(239)$ & $299^{\dagger}$ & $89^{\dagger}$ & $49^{\S}(30)$ & -** & - ${ }^{* *}$ \\
\hline \multicolumn{7}{|l|}{ Illinois } \\
\hline No. deaths (rate) & $1,398(10.4)$ & $1,110(17.1)$ & $288(4.2)$ & $792(6.2)$ & $679(10.6)$ & $113(1.8)$ \\
\hline Costs, million USD (per capita, USD*) & $1,780(138)$ & 1,474 & 304 & $1,409(109)$ & 1,307 & 123 \\
\hline \multicolumn{7}{|l|}{ Indiana } \\
\hline No. deaths (rate) & $948(14.3)$ & $756(23.4)$ & $192(5.6)$ & $364(5.7)$ & $290(9.0)$ & $74(2.3)$ \\
\hline Costs, million USD (per capita, USD*) & $1,210(183)$ & 1,023 & 194 & $597(90)$ & 515 & $86^{\dagger}$ \\
\hline \multicolumn{7}{|l|}{ lowa } \\
\hline No. deaths (rate) & $407(12.8)$ & $327(20.7)$ & $80(5.2)$ & $78(2.5)$ & $50(3.2)$ & $28(1.8)$ \\
\hline Costs, million USD (per capita, USD*) & $520(167)$ & 437 & $81^{\dagger}$ & $114(37)$ & $87^{\dagger}$ & $32^{\S}$ \\
\hline \multicolumn{7}{|l|}{ Kansas } \\
\hline No. deaths (rate) & $455(15.7)$ & $356(25.0)$ & $99(6.6)$ & $104(3.6)$ & $75(5.2)$ & $29(2.1)$ \\
\hline Costs, million USD (per capita, USD*) & $624(215)$ & 511 & $111^{\dagger}$ & $168(58)$ & 132 & 34 \\
\hline \multicolumn{7}{|l|}{ Kentucky } \\
\hline No. deaths (rate) & 727 (15.9) & $582(26.2)$ & $145(6.2)$ & $203(4.7)$ & $153(7.1)$ & $50(2.3)$ \\
\hline Costs, million USD (per capita, USD*) & $927(210)$ & 771 & 151 & $303(69)$ & 253 & $55^{\dagger}$ \\
\hline \multicolumn{7}{|l|}{ Louisiana } \\
\hline No. deaths (rate) & $679(14.3)$ & $506(22.2)$ & $173(7.0)$ & $538(11.6)$ & $428(18.6)$ & $110(4.7)$ \\
\hline Costs, million USD (per capita, USD*) & $888(191)$ & 692 & 176 & $941(202)$ & 796 & 135 \\
\hline \multicolumn{7}{|l|}{ Maine } \\
\hline No. deaths (rate) & $220(15.7)$ & $174(25.5)$ & $46(6.7)$ & $23(2.0)$ & $15(2.6)$ & $-^{\dagger \dagger}$ \\
\hline Costs, million USD (per capita, USD*) & $269^{\dagger}(202)$ & $219^{\dagger}$ & $49^{\dagger}$ & $35^{\S}(26)$ & -** & - $^{* *}$ \\
\hline Maryland & & & & & & \\
\hline No. deaths (rate) & $606(9.8)$ & $470(16.1)$ & $136(4.2)$ & $387(6.6)$ & $312(10.8)$ & $75(2.4)$ \\
\hline Costs, million USD (per capita, USD*) & 763 (128) & 617 & $140^{\dagger}$ & $692(116)$ & 593 & $91^{\dagger}$ \\
\hline Massachusetts & & & & & & \\
\hline No. deaths (rate) & $596(8.3)$ & $472(13.6)$ & $124(3.4)$ & $110(1.6)$ & $91(2.7)$ & $19(0.5)$ \\
\hline Costs, million USD (per capita, USD*) & $782(116)$ & 657 & 126 & $197(29)$ & 176 & $24^{\dagger}$ \\
\hline Michigan & & & & & & \\
\hline No. deaths (rate) & $1,354(13.2)$ & $1,062(21.3)$ & $292(5.6)$ & $589(6.2)$ & $465(9.8)$ & $124(2.6)$ \\
\hline Costs, million USD (per capita, USD*) & $1,735(175)$ & 1,461 & 276 & $990(100)$ & 831 & 149 \\
\hline Minnesota & & & & & & \\
\hline No. deaths (rate) & $686(12.2)$ & $525(18.8)$ & $161(5.9)$ & $101(1.9)$ & $69(2.6)$ & $32(1.2)$ \\
\hline Costs, million USD (per capita, USD*) & $914(168)$ & 741 & 172 & $170(31)$ & 125 & $40^{\dagger}$ \\
\hline Mississippi & & & & & & \\
\hline No. deaths (rate) & $380(12.5)$ & $299(20.8)$ & $81(5.3)$ & $332(11.3)$ & $277(19.4)$ & $55(3.5)$ \\
\hline Costs, million USD (per capita, USD*) & $481(161)$ & 406 & $74^{\dagger}$ & $530(177)$ & 484 & $62^{\dagger}$ \\
\hline Missouri & & & & & & \\
\hline No. deaths (rate) & $1,017(16.3)$ & $817(27.2)$ & $200(6.3)$ & $441(7.5)$ & $357(12.3)$ & $84(2.8)$ \\
\hline Costs, million USD (per capita, USD*) & $1,302(215)$ & 1,091 & 205 & $745(123)$ & 650 & 94 \\
\hline Montana & & & & & & \\
\hline No. deaths (rate) & $251(23.8)$ & $197(36.8)$ & $54(11.4)$ & $30(2.9)$ & $23(4.4)$ & $-{ }^{\dagger \dagger}$ \\
\hline Costs, million USD (per capita, USD*) & $302^{\dagger}(295)$ & $250^{\dagger}$ & $52^{\dagger}$ & $40^{\dagger}(39)$ & - ** & -** \\
\hline Nebraska & & & & & & \\
\hline No. deaths (rate) & $251(13.4)$ & $202(21.7)$ & $49(5.4)$ & $63(3.4)$ & $47(5.0)$ & $16(1.7)$ \\
\hline Costs, million USD (per capita, USD*) & $313(166)$ & 263 & $51^{+}$ & $108(58)$ & $91^{+}$ & —** \\
\hline Nevada & & & & & & \\
\hline No. deaths (rate) & $573(19.5)$ & $449(31.2)$ & $124(8.2)$ & $176(6.3)$ & $138(9.8)$ & $38(2.7)$ \\
\hline Costs, million USD (per capita, USD*) & $669(236)$ & 547 & $124^{\dagger}$ & $266(94)$ & 235 & $41^{\S}$ \\
\hline New Hampshire & & & & & & \\
\hline No. deaths (rate) & $247(17.6)$ & $191(27.5)$ & $56(8.1)$ & $17(1.3)^{9}$ & $-^{\dagger+}$ & - $^{\dagger+}$ \\
\hline Costs, million USD (per capita, USD*) & $302^{\dagger}(228)$ & $251^{\dagger}$ & $49^{\dagger}$ & - ${ }^{* *}$ & - ${ }^{* *}$ & - $^{* *}$ \\
\hline
\end{tabular}

See table footnotes on page 11. 
TABLE 2. (Continued) Suicide and homicide deaths, rates per 100,000 population, lifetime medical and work-loss costs, and lifetime medical and work-loss costs per capita, by state — United States, 2014

\begin{tabular}{|c|c|c|c|c|c|c|}
\hline \multirow[b]{3}{*}{ State } & \multicolumn{3}{|c|}{ Suicides } & \multicolumn{3}{|c|}{ Homicides } \\
\hline & \multirow[b]{2}{*}{ Total } & \multicolumn{2}{|c|}{ Sex } & \multirow[b]{2}{*}{ Total } & \multicolumn{2}{|c|}{ Sex } \\
\hline & & Male & Female & & Male & Female \\
\hline \multicolumn{7}{|l|}{ New Jersey } \\
\hline No. deaths (rate) & $786(8.3)$ & $590(12.9)$ & $196(4.1)$ & $372(4.4)$ & $302(7.2)$ & $70(1.6)$ \\
\hline Costs, million USD (per capita, USD*) & $958(107)$ & 748 & 203 & $654(73)$ & 568 & $80^{+}$ \\
\hline \multicolumn{7}{|l|}{ New Mexico } \\
\hline No. deaths (rate) & $449(21.0)$ & $350(33.4)$ & $99(9.2)$ & $135(6.8)$ & $106(10.5)$ & $29(2.9)$ \\
\hline Costs, million USD (per capita, USD*) & $594(285)$ & 501 & 98 & $218(105)$ & 183 & $32^{\S}$ \\
\hline \multicolumn{7}{|l|}{ New York } \\
\hline No. deaths (rate) & $1,700(8.1)$ & $1,262(12.5)$ & $438(4.0)$ & $662(3.3)$ & $536(5.5)$ & $126(1.2)$ \\
\hline Costs, million USD (per capita, USD*) & $2,139(108)$ & 1,674 & 435 & $1,157(59)$ & 1,010 & 147 \\
\hline \multicolumn{7}{|l|}{ North Carolina } \\
\hline No. deaths (rate) & $1,351(13.0)$ & $984(19.8)$ & $367(6.9)$ & $551(5.6)$ & $435(8.9)$ & $116(2.3)$ \\
\hline Costs, million USD (per capita, USD*) & $1,685(169)$ & 1,296 & 369 & $730(73)$ & 769 & 128 \\
\hline \multicolumn{7}{|l|}{ North Dakota } \\
\hline No. deaths (rate) & $137(17.5)$ & $113(27.8)$ & $24(6.7)$ & $15(2.0)^{\uparrow}$ & $13(3.0)$ & - $^{\dagger+}$ \\
\hline Costs, million USD (per capita, USD*) & $195^{\dagger}(264)$ & $169^{\dagger}$ & 一** & 一** & -** & -** \\
\hline \multicolumn{7}{|l|}{ Ohio } \\
\hline No. deaths (rate) & $1,491(12.6)$ & $1,163(20.1)$ & $328(5.7)$ & $578(5.2)$ & $472(8.4)$ & $106(1.9)$ \\
\hline Costs, million USD (per capita, USD*) & $1,939(167)$ & 1,588 & 344 & $955(82)$ & 843 & 122 \\
\hline \multicolumn{7}{|l|}{ Oklahoma } \\
\hline No. deaths (rate) & $736(19.1)$ & $561(29.5)$ & $175(9.2)$ & $250(6.5)$ & $183(9.5)$ & $67(3.5)$ \\
\hline Costs, million USD (per capita, USD*) & $999(258)$ & 801 & 186 & $409(105)$ & 316 & $83^{\dagger}$ \\
\hline \multicolumn{7}{|l|}{ Oregon } \\
\hline No. deaths (rate) & $782(18.7)$ & $614(30.1)$ & $168(7.9)$ & $99(2.4)$ & $65(3.1)$ & $34(1.7)$ \\
\hline Costs, million USD (per capita, USD*) & $911(229)$ & 755 & $157^{\dagger}$ & $131(33)$ & $104^{+}$ & $33^{+}$ \\
\hline \multicolumn{7}{|l|}{ Pennsylvania } \\
\hline No. deaths (rate) & $1,817(13.3)$ & $1,440(21.6)$ & $377(5.6)$ & $620(5.1)$ & $492(8.1)$ & $128(2.0)$ \\
\hline Costs, million USD (per capita, USD*) & $2,307(180)$ & 1,928 & 378 & $1,059(83)$ & 901 & 149 \\
\hline Rhode Island & & & & & & \\
\hline No. deaths (rate) & $113(10.0)$ & $82(14.9)$ & $31(5.4)$ & $27(2.5)$ & $23(4.2)$ & - $^{\dagger+}$ \\
\hline Costs, million USD (per capita, USD*) & $159^{\dagger}(151)$ & $120^{\dagger}$ & - $^{* *}$ & $45^{\dagger}(43)$ & - ** & - $^{* *}$ \\
\hline South Carolina & & & & & & \\
\hline No. deaths (rate) & $753(15.1)$ & $579(24.4)$ & $174(6.8)$ & $363(7.5)$ & $286(12.1)$ & $77(3.1)$ \\
\hline Costs, million USD (per capita, USD*) & $953(197)$ & 785 & 170 & $587(121)$ & 503 & $84^{\dagger}$ \\
\hline South Dakota & & & & & & \\
\hline No. deaths (rate) & $141(17.0)$ & $109(25.9)$ & $32(7.9)$ & $26(3.2)$ & $15(3.6)$ & $11(2.7)$ \\
\hline Costs, million USD (per capita, USD*) & $197^{\dagger}(231)$ & $162^{\dagger}$ & $37^{\dagger}$ & - ** & —** & —** \\
\hline Tennessee & & & & & & \\
\hline No. deaths (rate) & $948(14.1)$ & $746(23.3)$ & $202(5.8)$ & $379(5.9)$ & $309(9.6)$ & $70(2.2)$ \\
\hline Costs, million USD (per capita, USD*) & $1,241(189)$ & 1,032 & 214 & $595(91)$ & 523 & $82^{\dagger}$ \\
\hline Texas & & & & & & \\
\hline No. deaths (rate) & $3,254(12.2)$ & $2,528(19.5)$ & $726(5.4)$ & $1,389(5.1)$ & $1,059(7.8)$ & $330(2.5)$ \\
\hline Costs, million USD (per capita, USD*) & $4,264(158)$ & 3,490 & 754 & $2,240(83)$ & 1,867 & 386 \\
\hline Utah & & & & & & \\
\hline No. deaths (rate) & $559(20.6)$ & $418(31.0)$ & $141(10.5)$ & $61(2.1)$ & $39(2.7)$ & $22(1.4)$ \\
\hline Costs, million USD (per capita, USD*) & $802(273)$ & 634 & $158^{\dagger}$ & $89^{\dagger}(30)$ & $67^{\dagger}$ & $25^{+}$ \\
\hline Vermont & & & & & & \\
\hline No. deaths (rate) & $124(18.6)$ & $102(30.7)$ & $22(7.2)$ & $16(2.9)^{9}$ & $13(4.8)$ & $-^{\dagger+}$ \\
\hline Costs, million USD (per capita, USD*) & $148^{\dagger}(237)$ & $131^{\dagger}$ & 一** & -** & 一** & 一** \\
\hline Virginia & & & & & & \\
\hline No. deaths (rate) & $1,122(12.9)$ & $870(20.7)$ & $252(5.7)$ & $339(4.1)$ & $249(5.9)$ & $90(2.2)$ \\
\hline Costs, million USD (per capita, USD*) & $1,412(170)$ & 1,150 & 252 & $555(67)$ & 449 & $105^{\dagger}$ \\
\hline Washington & & & & & & \\
\hline No. deaths (rate) & $1,119(15.2)$ & $854(23.5)$ & $265(7.2)$ & $211(3.0)$ & $157(4.4)$ & $54(1.6)$ \\
\hline Costs, million USD (per capita, USD*) & $1,404(199)$ & 1,147 & 253 & $333(47)$ & 272 & $63^{\dagger}$ \\
\hline West Virginia & & & & & & \\
\hline No. deaths (rate) & 359 (18.1) & $280(28.6)$ & $79(8.1)$ & $103(5.9)$ & $70(7.9)$ & $33(3.9)$ \\
\hline Costs, million USD (per capita, USD*) & $426(230)$ & 346 & $71^{\dagger}$ & $156(85)$ & $113^{\dagger}$ & $41^{\dagger}$ \\
\hline
\end{tabular}

See table footnotes on page 11 . 
TABLE 2. (Continued) Suicide and homicide deaths, rates per 100,000 population, lifetime medical and work-loss costs, and lifetime medical and work-loss costs per capita, by state — United States, 2014

\begin{tabular}{|c|c|c|c|c|c|c|}
\hline \multirow[b]{3}{*}{ State } & \multicolumn{3}{|c|}{ Suicides } & \multicolumn{3}{|c|}{ Homicides } \\
\hline & \multirow[b]{2}{*}{ Total } & \multicolumn{2}{|c|}{ Sex } & \multirow[b]{2}{*}{ Total } & \multicolumn{2}{|c|}{ Sex } \\
\hline & & Male & Female & & Male & Female \\
\hline \multicolumn{7}{|l|}{ Wisconsin } \\
\hline No. deaths (rate) & $769(13.1)$ & $598(20.6)$ & $171(5.9)$ & $166(3.0)$ & $126(4.5)$ & $40(1.4)$ \\
\hline Costs, million USD (per capita, USD*) & $981(170)$ & 806 & 170 & $274(48)$ & 227 & $45^{\dagger}$ \\
\hline \multicolumn{7}{|l|}{ Wyoming } \\
\hline No. deaths (rate) & $120(20.7)$ & $96(32.3)$ & $24(8.7)$ & $24(4.4)$ & $16(5.8)$ & - \\
\hline Costs, million USD (per capita, USD*) & $153^{\dagger}(262)$ & $131^{\dagger}$ & $21^{\dagger}$ & —* & —** & —** \\
\hline
\end{tabular}

* Costs per capita calculated only for totals.

+ Average medical cost was statistically unstable; however, it accounted for less than $1 \%$ of combined average cost.

$\S$ Average medical cost was statistically unstable; however, it accounted for less than $5 \%$ of combined average cost.

I Rates based on $\leq 20$ deaths might be unstable.

** Both average medical cost and average work loss cost were statistically unstable.

t+ State-level counts and rates based on $<10$ deaths have been suppressed. 